Proceedings of the International Congress of Mathematicians

Hyderabad, India, 2010

\title{
Discrete Complex Analysis and Probability
}

\author{
Stanislav Smirnov *
}

\begin{abstract}
We discuss possible discretizations of complex analysis and some of their applications to probability and mathematical physics, following our recent work with Dmitry Chelkak, Hugo Duminil-Copin and Clément Hongler.
\end{abstract}

Mathematics Subject Classification (2000). Primary 30G25; Secondary 05C81, $60 \mathrm{~K} 35,81 \mathrm{~T} 40,82 \mathrm{~B} 20$.

Keywords. Discrete complex analysis, discrete analytic function, Ising model, selfavoiding walk, conformal invariance

\section{Introduction}

The goal of this note is to discuss some of the applications of discrete complex analysis to problems in probability and statistical physics. It is not an exhaustive survey, and it lacks many references. Forgoing completeness, we try to give a taste of the subject through examples, concentrating on a few of our recent papers with Dmitry Chelkak, Hugo Duminil-Copin and Clément Hongler CS08, CS09, CS10, DCS10, HS10. There are certainly other interesting developments in discrete complex analysis, and it would be a worthy goal to write an extensive exposition with an all-encompassing bibliography, which we do not attempt here for lack of space.

Complex analysis (we restrict ourselves to the case of one complex or equivalently two real dimensions) studies analytic functions on (subdomains of) the complex plane, or more generally analytic structures on two dimensional manifolds. Several things are special about the (real) dimension two, and we won't discuss an interesting and often debated question, why exactly complex analysis is so nice and elegant. In particular, several definitions lead to identical class of analytic functions, and historically different adjectives (regular, analytic, holomorphic, monogenic) were used, depending on the context. For example, an analytic function has a local power series expansion around every point, while a holomorphic function has a complex derivative at every point. Equivalence of these definitions

*This research was supported by the European Research Council AG "CONFRA" and by the Swiss National Science Foundation. We would like to thank Dmitry Chelkak for comments on the preliminary version of this paper. 
is a major theorem in complex analysis, and there are many other equivalent definitions in terms of Cauchy-Riemann equations, contour integrals, primitive functions, hydrodynamic interpretation, etc. Holomorphic functions have many nice properties, and hundreds of books were devoted to their study.

Consider now a discretized version of the complex plane: some graph embedded into it, say a square or triangular lattice (more generally one can speak of discretizations of Riemann surfaces). Can one define analytic functions on such a graph? Some of the definitions do not admit a straightforward discretization: e.g. local power series expansions do not make sense on a lattice, so we cannot really speak of discrete analyticity. On the other hand, as soon as we define discrete derivatives, we can ask for the holomorphicity condition. Thus it is philosophically more correct to speak of discrete holomorphic, rather than discrete analytic functions. We will use the term preholomorphic introduced by Ferrand Fer44, as we prefer it to the term monodiffric used by Isaacs in the original papers Isa41, Isa52. (a play on the term monogenic used by Cauchy for continuous analytic functions).

Though the preholomorphic functions are easy to define, there is a lack of expository literature about them. We see two main reasons: firstly, there is no canonical preholomorphicity definition, and one can argue which of the competing approaches is better (the answer probably depends on potential applications). Secondly, it is straightforward to transfer to the discrete case beginnings of the usual complex analysis (a nice topic for an undergraduate research project), but the easy life ends when it becomes necessary to multiply preholomorphic functions. There is no easy and natural way to proceed and the difficulty is addressed depending on the problem at hand.

As there seems to be no canonical discretization of the complex analysis, we would rather adopt a utilitarian approach, working with definitions corresponding to interesting objects of probabilistic origin, and allowing for a passage to the scaling limit. We want to emphasize, that we are concerned with the following triplets:

1. A planar graph,

2. Its embedding into the complex plane,

\section{Discrete Cauchy-Riemann equations.}

We are interested in triplets such that the discrete complex analysis approximates the continuous one. Note that one can start with only a few elements of the triplet, which gives some freedom. For example, given an embedded graph, one can ask which discrete difference equations have solutions close to holomorphic functions. Or, given a planar graph and a notion of preholomorphicity, one can look for an appropriate embedding.

The ultimate goal is to find lattice models of statistical physics with preholomorphic observables. Since those observables would approximate holomorphic functions, some information about the original model could be subsequently deduced. 
Below we start with several possible definitions of the preholomorphic functions along with historical remarks. Then we discuss some of their recent applications in probability and statistical physics.

\section{Discrete holomorphic functions}

For a given planar graph, there are several ways to define preholomorphic functions, and it is not always clear which way is preferable. A much better known class is that of discrete harmonic (or preharmonic) functions, which can be defined on any graph (not necessarily planar), and also in more than one way. However, one definition stands out as the simplest: a function on the vertices of graph is said to be preharmonic at a vertex $v$, if its discrete Laplacian vanishes:

$$
0=\Delta H(u):=\sum_{v: \text { neighbor of } u}(H(v)-H(u)) .
$$

More generally, one can put weights on the edges, which would amount to taking different resistances in the electric interpretation below. Preharmonic functions on planar graphs are closely related to discrete holomorphicity: for example, their gradients defined on the oriented edges by

$$
F(\overrightarrow{u v}):=H(v)-H(u)
$$

are preholomorphic. Note that the edge function above is antisymmetric, i.e. $F(\overrightarrow{u v})=-F(\overrightarrow{v u})$.

Both classes with the definitions as above are implicit already in the 1847 work of Kirchhoff [Kir47, who interpreted a function defined on oriented edges as an electric current flowing through the graph. If we assume that all edges have unit resistance, than the sum of currents flowing from a vertex is zero by the first Kirchhoff law:

$$
\sum_{u: \text { neighbor of } v} F(\overrightarrow{u v})=0,
$$

and the sum of the currents around any oriented closed contour $\gamma$ (for the planar graphs it is sufficient to consider contours around faces) face is zero by the second Kirchhoff law:

$$
\sum_{\overrightarrow{u v} \in \gamma} F(\overrightarrow{u v})=0
$$

The two laws are equivalent to saying that $F$ is given by the gradient of a potential function $H$ as in (2), and the latter function is preharmonic (11). One can equivalently think of a hydrodynamic interpretation, with $F$ representing the flow of liquid. Then conditions (3) and (4) mean that the flow is divergence- and curl-free correspondingly. Note that in the continuous setting similarly defined gradients of harmonic functions on planar domains coincide up to complex conjugation with

holomorphic functions. And in higher dimensions harmonic gradients were proposed as one of their possible generalizations. 
There are many other ways to introduce discrete structures on graphs, which can be developed in parallel to the usual complex analysis. We have in mind mostly such discretizations that restrictions of holomorphic (or harmonic) functions become approximately preholomorphic (or preharmonic). Thus we speak about graphs embedded into the complex plane or a Riemann surface, and the choice of embedding plays an important role. Moreover, the applications we are after require passages to the scaling limit (as mesh of the lattice tends to zero), so we want to deal with discrete structures which converge to the usual complex analysis as we take finer and finer graphs.

Preharmonic functions satisfying (1) on the square lattices with decreasing mesh fit well into this philosophy, and were studied in a number of papers in early twentieth century (see e.g. PW23, Bou26, Lus26]), culminating in the seminal work of Courant, Friedrichs and Lewy. It was shown in CFL28 that solution to the Dirichlet problem for a discretization of an elliptic operator converges to the solution of the analogous continuous problem as the mesh of the lattice tends to zero. In particular, a preharmonic function with given boundary values converges in the scaling limit to a harmonic function with the same boundary values in a rather strong sense, including convergence of all partial derivatives.

Preholomorphic functions distinctively appeared for the first time in the papers Isa41, Isa52 of Isaacs, where he proposed two definitions (and called such functions "monodiffric"). A few papers of his and others followed, studying the first definition (5), which is asymmetric on the square lattice. More recently the first definition was studied by Dynnikov and Novikov DN03 in the triangular lattice context, where it becomes symmetric (the triangular lattice is obtained from the square lattice by adding all the diagonals in one direction).

The second, symmetric, definition was reintroduced by Ferrand, who also discussed the passage to the scaling limit Fer44, LF55]. This was followed by extensive studies of Duffin and others, starting with Duf56.

Both definitions ask for a discrete version of the Cauchy-Riemann equations $\partial_{i \alpha} F=i \partial_{\alpha} F$ or equivalently that $z$-derivative is independent of direction. Consider a subregion $\Omega_{\epsilon}$ of the mesh $\epsilon$ square lattice $\epsilon \mathbb{Z}^{2} \subset \mathbb{C}$ and define a function on its vertices. Isaacs proposed the following two definitions, replacing the derivatives by discrete differences. His "monodiffric functions of the first kind" are required to satisfy inside $\Omega_{\epsilon}$ the following identity:

$$
F(z+i \epsilon)-F(z)=i(F(z+\epsilon)-F(z))
$$

which can be rewritten as

$$
\frac{F(z+i \epsilon)-F(z)}{(z+i \epsilon)-z}=\frac{F(z+\epsilon)-F(z)}{(z+\epsilon)-z} .
$$

We will be working with his second definition, which is more symmetric and also appears naturally in probabilistic context (but otherwise the theories based on two definitions are almost the same). We say that a function is preholomorphic, if inside $\Omega_{\epsilon}$ it satisfies the following identity, illustrated in Figure 1

$$
F(z+i \epsilon)-F(z+\epsilon)=i(F(z+\epsilon(1+i))-F(z))
$$



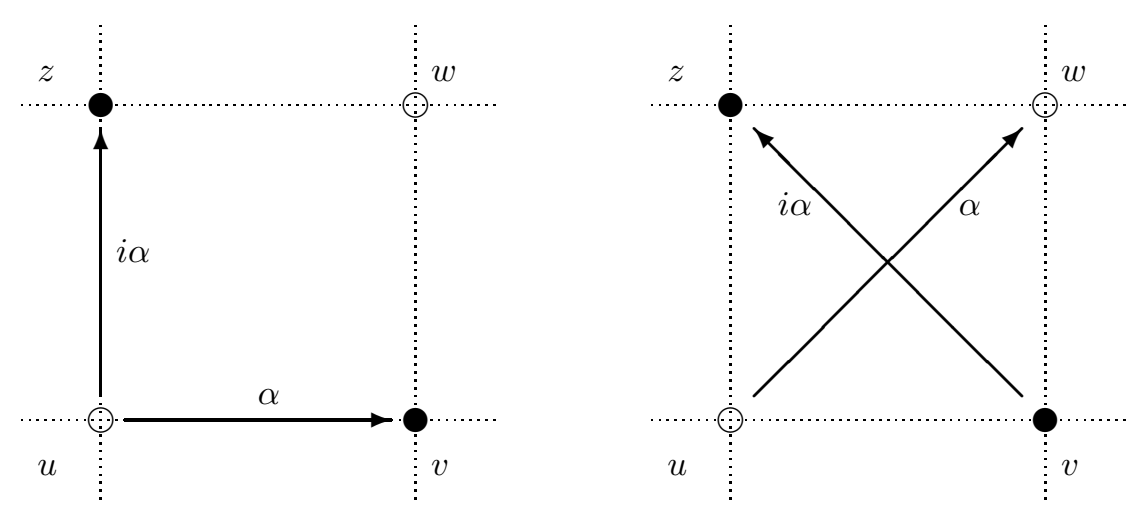

Figure 1. The first and the second Isaacs' definitions of discrete holomorphic functions: multiplied by $i$ difference along the vector $\alpha$ is equal to the difference along the rotated vector $i \alpha$. Note that the second definition (on the right) is symmetric with respect to lattice rotations, while the first one is not.

which can also be rewritten as

$$
\frac{F(z+i \epsilon)-F(z+\epsilon)}{(z+i \epsilon)-(z+\epsilon)}=\frac{F(z+\epsilon(1+i))-F(z)}{(z+\epsilon(1+i))-z} .
$$

It is easy to see that restrictions of continuous holomorphic functions to the mesh $\epsilon$ square lattice satisfy this identity up to $O\left(\epsilon^{3}\right)$. Note also that if we color the lattice in the chess-board fashion, the complex identity (6) can be written as two real identities (its real and imaginary parts), one involving the real part of $F$ at black vertices and the imaginary part of $F$ at white vertices, the other one - vice versa. So unless we have special boundary conditions, $F$ splits into two "demifunctions" (real at white and imaginary at black vs. imaginary at black and real at white vertices), and some prefer to consider just one of those, i.e. ask $F$ to be purely real at black vertices and purely imaginary at white ones.

The theory of so defined preholomorphic functions starts much like the usual complex analysis. It is easy to check, that for preholomorphic functions sums are also preholomorphic, discrete contour integrals vanish, primitive (in a simplyconnected domain) and derivative are well-defined and are preholomorphic functions on the dual square lattice, real and imaginary parts are preharmonic on their respective black and white sublattices, etc. Unfortunately, the product of two preholomorphic functions is no longer preholomorphic: e.g., while restrictions of $1, z$, and $z^{2}$ to the square lattice are preholomorphic, the higher powers are only approximately so.

Situation with other possible definitions is similar, with much of the linear complex analysis being easy to reproduce, and problems appearing when one has 
to multiply preholomorphic functions. Pointwise multiplication cannot be consistently defined, and though one can introduce convolution-type multiplication, the possible constructions are non-local and cumbersome. Sometimes, for different graphs and definitions, problems appear even earlier, with the first derivative not being preholomorphic.

Our main reason for choosing the definition (6) is that it naturally appears in probabilistic context. It was also noticed by Duffin that (6) nicely generalizes to a larger family of rhombic lattices, where all the faces are rhombi. Equivalently, one can speak of isoradial graphs, where all faces are inscribed into circles of the same radius - an isoradial graph together with its dual forms a rhombic lattice.

There are two main reasons to study this particular family. First, this is perhaps the largest family of graphs for which the Cauchy-Riemann operator admits a nice discretization. Indeed, restrictions of holomorphic functions to such graphs are preholomorphic to higher orders. This was the reason for the introduction of complex analysis on rhombic lattices by Duffin Duf68 in late sixties. More recently, the complex analysis on such graphs was studied for the sake of probabilistic applications [Mer01, Ken02, CS08.

On the other hand, this seems to be the largest family where certain lattice models, including the Ising model, have nice integrability properties. In particular, the critical point can be defined with weights depending only on the local structure, and the star-triangle relation works out nicely. It seems that the first appearance of related family of graphs in the probabilistic context was in the work of Baxter Bax78, where the eight vertex and Ising models were considered on $Z$-invariant graphs, arising from planar line arrangements. These graphs are topologically the same as the isoradial ones, and though they are embedded differently into the plane, by KS05 they always admit isoradial embeddings. In Bax78 Baxter was not passing to the scaling limit, and so the actual choice of embedding was immaterial for his results. However, his choice of weights in the models would suggest an isoradial embedding, and the Ising model was so considered by Mercat [Mer01, Boutilier and de Tilière [BdT08, BdT09, Chelkak and the author CS09. Additionally, the dimer and the uniform spanning tree models on such graphs also have nice properties, see e.g. Ken02.

We would also like to remark that rhombic lattices form a rather large family of graphs. While not every topological quadrangulation (graph all of whose faces are quadrangles) admits a rhombic embedding, Kenyon and Schlenker [KS05] gave a simple topological condition necessary and sufficient for its existence.

So this seems to be the most general family of graphs appropriate for our subject, and most of what we discuss below generalizes to it (though for simplicity we speak of the square and hexagonal lattices only).

\section{Applications of preholomorphic functions}

Besides being interesting in themselves, preholomorphic functions found several diverse applications in combinatorics, analysis, geometry, probability and physics. 
After the original work of Kirchhoff, the first notable application was perhaps the famous article [BSST40] of Brooks, Smith, Stone and Tutte, who used preholomorphic functions to construct tilings of rectangles by squares.

Several applications to analysis followed, starting with a new proof of the Riemann uniformization theorem by Ferrand LF55. Solving the discrete version of the usual minimization problem, it is immediate to establish the existence of the minimizer and its properties, and then one shows that it has a scaling limit, which is the desired uniformization. Duffin and his co-authors found a number of similar applications, including construction of the Bergman kernel by Dieter and Mastin [DM71. There were also studies of discrete versions of the multi-dimensional complex analysis, see e.g. Kiselman's [Kis05].

In Thu86] Thurston proposed circle packings as another discretization of complex analysis. They found some beautiful applications, including yet another proof of the Riemann uniformization theorem by Rodin and Sullivan [RS87. More interestingly, they were used by He and Schramm HS93 in the best result so far on the Koebe uniformization conjecture, stating that any domain can be conformally uniformized to a domain bounded by circles and points. In particular, they established the conjecture for domains with countably many boundary components. More about circle packings can be learned form Stephenson's book Ste05. Note that unlike the discretizations discussed above, the circle packings lead to non-linear versions of the Cauchy-Riemann equations, see e.g. the discussion in [BMS05.

There are other interesting applications to geometry, analysis, combinatorics, probability, and we refer the interested reader to the expositions by Lovász [Lov04, Stephenson [Ste05, Mercat Mer07, Bobenko and Suris [BS08].

In this note we are interested in applications to probability and statistical physics. Already the Kirchhoff's paper Kir47. makes connection between the Uniform Spanning Tree and preharmonic (and so preholomorphic) functions.

Connection of Random Walk to preharmonic functions was certainly known to many researchers in early twentieth century, and figured implicitly in many papers. It is explicitly discussed by Courant, Friedrichs and Lewy in CFL28, with preharmonic functions appearing as Green's functions and exit probabilities for the Random Walk.

More recently, Kenyon found preholomorphic functions in the dimer model (and in the Uniform Spanning Tree in a way different from the original considerations of Kirchhoff). He was able to obtain many beautiful results about statistics of the dimer tilings, and in particular, showed that those have a conformally invariant scaling limit, described by the Gaussian Free Field, see Ken00, Ken01. More about Kenyon's results can be found in his expositions Ken04, Ken09. An approximately preholomorphic function was found by the author in the critical site percolation on the triangular lattice, allowing to prove the Cardy's formula for crossing probabilities Smi01b, Smi01a.

Finally, we remark that various other discrete relations were observed in many integrable two dimensional models of statistical physics, but usually no explicit connection was made with complex analysis, and no scaling limit was considered. 
Here we are interested in applications of integrability parallel to that for the Random Walk and the dimer model above. Namely, once a preholomorphic function is observed in some probabilistic model, we can pass to the scaling limit, obtaining a holomorphic function. Thus, the preholomorphic observable is approximately equal to the limiting holomorphic function, providing some knowledge about the model at hand. Below we discuss applications of this philosophy, starting with the Ising model.

\section{The Ising model}

In this Section we discuss some of the ways how preholomorphic functions appear in the Ising model at criticality. The observable below was proposed in Smi06 for the hexagonal lattice, along with a possible generalization to $O(N)$ model. Similar objects appeared earlier in Kadanoff and Ceva KC71 and in Mercat Mer01, though boundary values and conformal covariance, which are central to us, were never discussed.

The scaling limit and properties of our observable on isoradial graphs were worked out by Chelkak and the author in CS09. It is more appropriate to consider it as a fermion or a spinor, by writing $F(z) \sqrt{d z}$, and with more general setup one has to proceed in this way.

Earlier we constructed a similar fermion for the random cluster representation of the Ising model, see Smi06, Smi10] and our joint work with Chelkak CS09] for generalization to isoradial graphs (and also independent work of Riva and Cardy [RC06] for its physical connections). It has a simpler probabilistic interpretation than the fermion in the spin representation, as it can be written as the probability of the interface between two marked boundary points passing through a point inside, corrected by a complex weight depending on the winding.

The fermion for the spin representation is more difficult to construct. Below we describe it in terms of contour collections with distinguished points. Alternatively it corresponds to the partition function of the Ising model with a $\sqrt{z}$ monodromy at a given edge, corrected by a complex weight; or to a product of order and disorder operators at neighboring site and dual site.

We will consider the Ising model on the mesh $\epsilon$ square lattice. Let $\Omega_{\epsilon}$ be a discretization of some bounded domain $\Omega \subset \mathbb{C}$. The Ising model on $\Omega_{\epsilon}$ has configurations $\sigma$ which assign \pm 1 (or simply \pm ) spins $\sigma(v)$ to vertices $v \in \Omega_{\epsilon}$ and Hamiltonian defined (in the absence of an external magnetic field) by

$$
H(\sigma)=-\sum_{\langle u, v\rangle} \sigma(u) \sigma(v),
$$

where the sum is taken over all edges $\langle u, v\rangle$ inside $\Omega_{\epsilon}$. Then the partition function is given by

$$
Z=\sum_{\sigma} \exp (-\beta H(\sigma))
$$



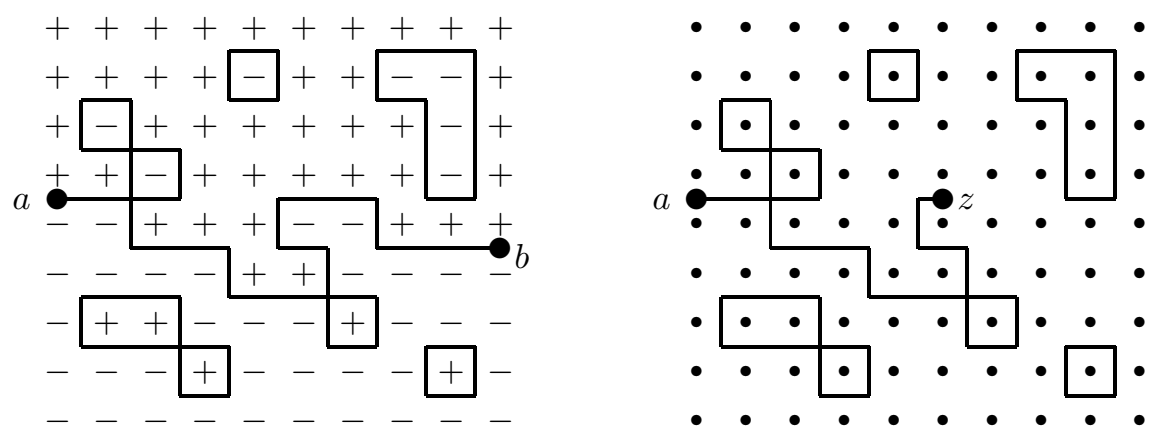

Figure 2. Left: configuration of spins in the Ising model with Dobrushin boundary conditions, its contour representation, and an interface between two boundary points. Right: an example of a configuration considered for the Fermionic observable: a number of loops and a contour connecting $a$ to $z$. It can be represented as a spin configuration with a monodromy at $z$.

and probability of a given spin configuration becomes

$$
\mathbb{P}(\sigma)=\exp (-\beta H(\sigma)) / Z .
$$

Here $\beta \geq 0$ is the temperature parameter (behaving like the reciprocal of the actual temperature), and Kramers and Wannier have established [KW41] that its critical value is given by $\beta_{c}=\log (\sqrt{2}+1) / 2$.

Now represent the spin configurations graphically by a collection of interfaces contours on the dual lattice, separating plus spins from minus spins, the so-called low-temperature expansion, see Figure 2] A contour collection is a set of edges, such that an even number emanates from every vertex. In such case the contours can be represented as a union of loops (possibly in a non-unique way, but we do not distinguish between different representations). Note that each contour collection corresponds to two spin collections which are negatives of each other, or to one if we fix the spin value at some vertex. The partition function of the Ising model can be rewritten in terms of the contour configurations $\omega$ as

$$
Z=\sum_{\omega} x^{\text {length of contours }}
$$

Each neighboring pair of opposite spins contributes an edge to the contours, and so a factor of $x=\exp (-2 \beta)$ to the partition function. Note that the critical value is $x_{c}=\exp \left(-2 \beta_{c}\right)=\sqrt{2}-1$.

We now want to define a preholomorphic observable. To this effect we need to distinguish at least one point (so that the domain has a non-trivial conformal modulus). One of the possible applications lies in relating interfaces to Schramm's SLE curves, in the simplest setup running between two boundary points. To obtain a discrete interface between two boundary points $a$ and $b$, we introduce Dobrushin boundary conditions: + on one boundary arc and - on another, see Figure 2 . Then those become unique points with an odd number of contour edges emanating from them. 

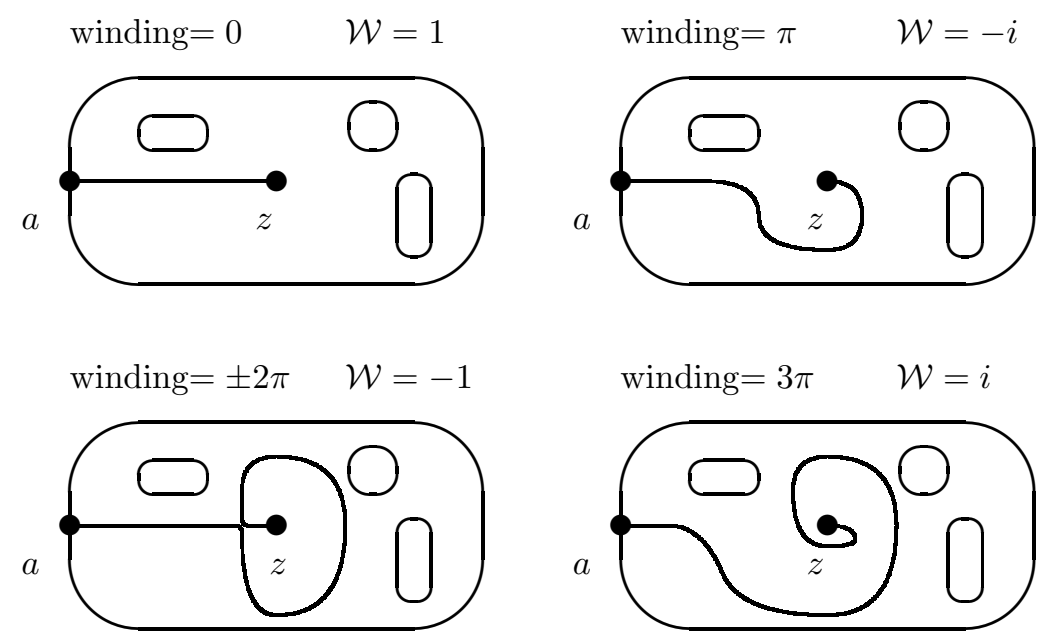

Figure 3. Examples of Fermionic weights one obtains depending on the winding of the interface. Note that in the bottom left example there are two ways to trace the interface from $a$ to $z$ without self-intersections, which give different windings $\pm 2 \pi$, but the same complex weight $\mathcal{W}=-1$.

Now to define our fermion, we allow the second endpoint of the interface to move inside the domain. Namely, take an edge center $z$ inside $\Omega_{\epsilon}$, and define

$$
F_{\epsilon}(z):=\sum_{\omega(a \rightarrow z)} x^{\text {length of contours }} \mathcal{W}(\omega(a \rightarrow z))
$$

where the sum is taken over all contour configurations $\omega=\omega(a \rightarrow z)$ which have two exceptional points: $a$ on the boundary and $z$ inside. So the contour collection can be represented (perhaps non-uniquely) as a collection of loops plus an interface between $a$ and $z$.

Furthermore, the sum is corrected by a Fermionic complex weight, depending on the configuration:

$$
\mathcal{W}(\omega(a \rightarrow z)):=\exp (-i s \text { winding }(\gamma, a \rightarrow z))
$$

Here the winding is the total turn of the interface $\gamma$ connecting $a$ to $z$, counted in radians, and the spin $s$ is equal to $1 / 2$ (it should not be confused with the Ising spins \pm 1 ). For some collections the interface can be chosen in more than one way, and then we trace it by taking a left turn whenever an ambiguity arises. Another choice might lead to a different value of winding, but if the loops and the interface have no "transversal" self-intersections, then the difference will be a multiple of $4 \pi$ and so the complex weight $\mathcal{W}$ is well-defined. Equivalently we can write

$$
\mathcal{W}(\omega(a \rightarrow z))=\lambda^{\# \text { signed turns of } \gamma}, \quad \lambda:=\exp \left(-i s \frac{\pi}{2}\right)
$$

see Figure 3 for weights corresponding to different windings. 


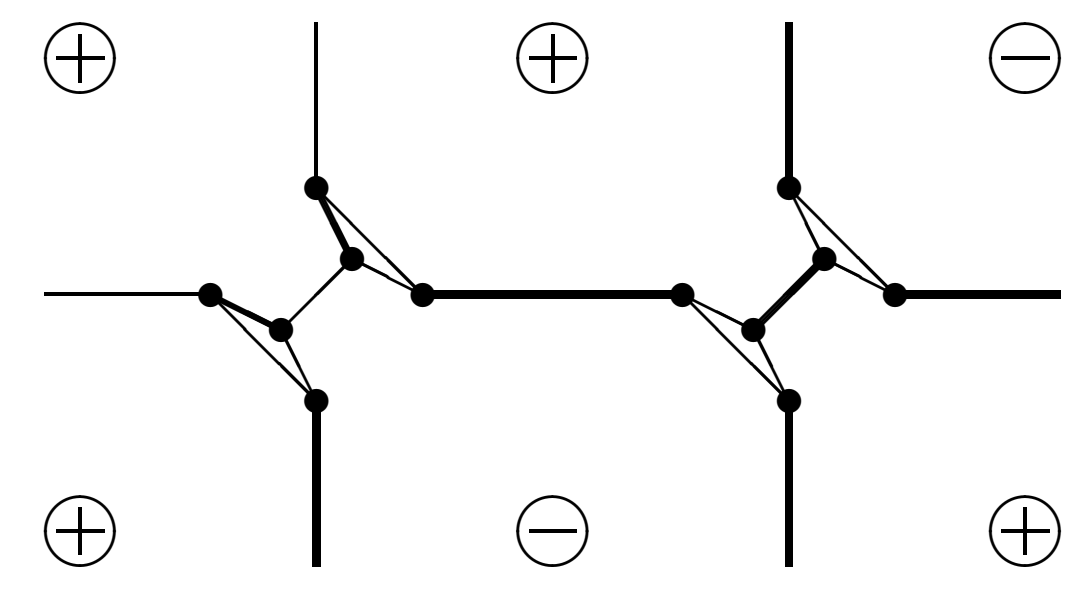

Figure 4. Fisher graph for a region of the square lattice, a spin configuration and a corresponding dimer configuration, with dimers represented by the bold edges.

Remark 1. Removing complex weight $\mathcal{W}$ one retrieves the correlation of spins on the dual lattice at the dual temperature $x^{*}$, a corollary of the Kramers-Wannier duality.

Remark 2. While such contour collections cannot be directly represented by spin configurations, one can obtain them by creating a disorder operator, i.e. a monodromy at $z$ : when one goes one time around $z$, spins change their signs.

Our first theorem is the following, which is proved for general isoradial graphs in CS09, with a shorter proof for the square lattice given in [CS10]:

Theorem 1 (Chelkak, Sminnov). For Ising model at criticality, $F$ is a preholomorphic solution of a Riemann boundary value problem. When mesh $\epsilon \rightarrow 0$,

$$
F_{\epsilon}(z) / \sqrt{\epsilon} \rightrightarrows \sqrt{P^{\prime}(z)} \text { inside } \Omega
$$

where $P$ is the complex Poisson kernel at a: a conformal map $\Omega \rightarrow \mathbb{C}_{+}$such that $a \mapsto \infty$. Here both sides should be normalized in the same chart around $b$.

Remark 3. For non-critical values of $x$ observable $F$ becomes massive preholomorphic, satisfying the discrete analogue of the massive Cauchy-Riemann equations: $\bar{\partial} F=i m\left(x-x_{c}\right) \bar{F}$, cf. MS09.

Remark 4. Ising model can be represented as a dimer model on the Fisher graph. For example, on the square lattice, one first represents the spin configuration as above - by the collection of contours on the dual lattice, separating + and spins. Then the dual lattice is modified with every vertex replaced by a "city" of six vertices, see Figure 4. It is easy to see that there is a natural bijection between 
contour configurations on the dual square lattice and dimer configuration on its Fisher graph.

Then, similarly to the work of Kenyon for the square lattice, the coupling function for the Fisher lattice will satisfy difference equations, which upon examination turn out to be another discretization of Cauchy-Riemann equations, with different projections of the preholomorphic function assigned to six vertices in a "city". One can then reinterpret the coupling function in terms of the Ising model, and this is the approach taken by Boutilier and de Tilière [BdT08, BdT09.

This is also how the author found the observable discussed in this Section, observing jointly with Kenyon in 2002 that it has the potential to imply the convergence of the interfaces to the Schramm's SLE curve.

The key to establishing Theorem 1 is the observation that the function $F$ is preholomorphic. Moreover, it turns out that $F$ satisfies a stronger form of preholomorphicity, which implies the usual one, but is better adapted to fermions.

Consider the function $F$ on the centers of edges. We say that $F$ is strongly (or spin) preholomorphic if for every centers $u$ and $v$ of two neighboring edges emanating from a vertex $w$, we have

$$
\operatorname{Proj}(F(v), 1 / \sqrt{\alpha})=\operatorname{Proj}(F(u), 1 / \sqrt{\alpha}),
$$

where $\alpha$ is the unit bisector of the angle $u w v$, and $\operatorname{Proj}(p, q)$ denotes the orthogonal projection of the vector $p$ on the vector $q$. Equivalently we can write

$$
F(v)+\bar{\alpha} \overline{F(v)}=F(u)+\bar{\alpha} \overline{F(u)} .
$$

This definition implies the classical one for the square lattice, and it also easily adapts to the isoradial graphs. Note that for convenience we assume that the interface starts from $a$ in the positive real direction as in Figure 2, which slightly changes weights compared to the convention in CS09.

The strong preholomorphicity of the Ising model fermion is proved by constructing a bijection between configurations included into $F(v)$ and $F(u)$. Indeed, erasing or adding half-edges $w u$ and $w v$ gives a bijection $\omega \leftrightarrow \tilde{\omega}$ between configuration collections $\{\omega(u)\}$ and $\{\omega(v)\}$, as illustrated in Figure 5. To check (8), it is sufficient to check that the sum of contributions from $\omega$ and $\tilde{\omega}$ satisfies it. Several possible configurations can be found, but essentially all boil down to the two illustrated in Figure 5

Plugging the contributions from Figure 5 into the equation (87), we are left to check the following two identities:

$$
\lambda+\lambda \bar{\lambda}=1+\lambda \overline{1}, \quad \lambda x+\lambda \overline{\lambda x}=\lambda^{2}+\lambda \bar{\lambda}^{2} .
$$

The first identity always holds, while the second one is easy to verify when $x=$ $x_{c}=\sqrt{2}-1$ and $\lambda=\exp (-\pi i / 4)$. Note that in our setup on the square lattice $\lambda$ (or the spin $s$ ) is already fixed by the requirement that the complex weight is well-defined, and so the second equation in (9) uniquely fixes the allowed value of $x$. In the next Section we will discuss a more general setup, allowing for different values of the spin, corresponding to other lattice models. 

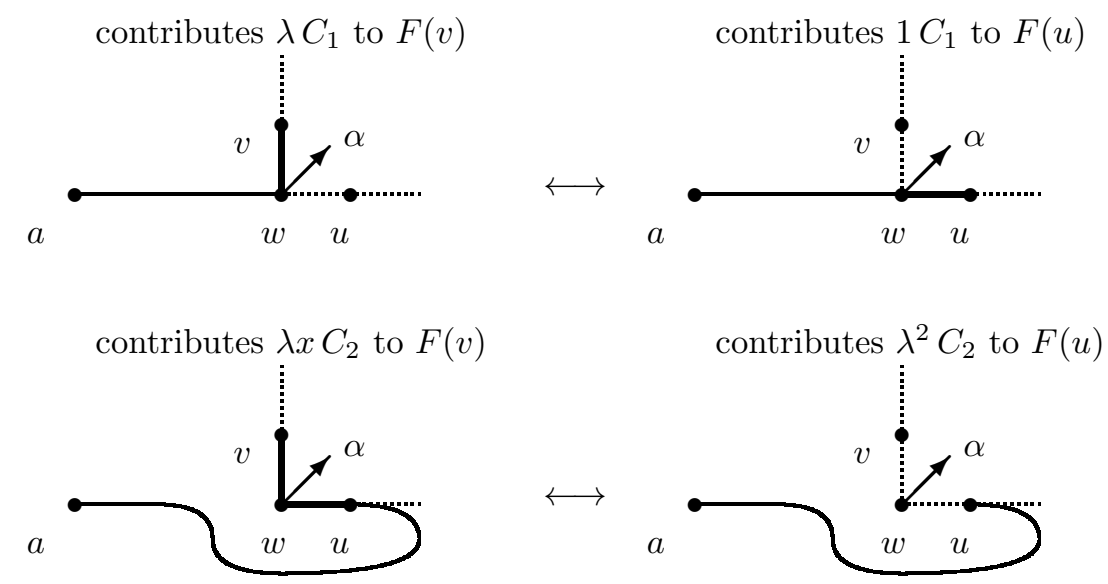

Figure 5. Involution on the Ising model configurations, which adds or erases half-edges $v w$ and $u w$. There are more pairs, but their relative contributions are always easy to calculate and each pair taken together satisfies the discrete Cauchy-Riemann equations. Note that with the chosen orientation constants $C_{1}$ and $C_{2}$ above are real.

To determine $F$ using its preholomorphicity, we need to understand its behavior on the boundary. When $z \in \partial \Omega_{\epsilon}$, the winding of the interface connecting $a$ to $z$ inside $\Omega_{\epsilon}$ is uniquely determined, and coincides with the winding of the boundary itself. This amounts to knowing $\operatorname{Arg}(F)$ on the boundary, which would be sufficient to determine $F$ knowing the singularity at $a$ or the normalization at $b$.

In the continuous setting the condition obtained is equivalent to the Riemann Boundary Value Problem (a homogeneous version of the Riemann-Hilbert-Privalov BVP)

$$
\operatorname{Im}\left(F(z) \cdot(\text { tangent to } \partial \Omega)^{1 / 2}\right)=0,
$$

with the square root appearing because of the Fermionic weight. Note that the homogeneous BVP above has conformally covariant solutions (as $\sqrt{d z}$-forms), and so is well defined even in domains with fractal boundaries. The Riemann BVP (10) is clearly solved by the function $\sqrt{P_{a}^{\prime}(z)}$, where $P$ is the Schwarz kernel at $a$ (the complex version of the Poisson kernel), i.e. a conformal map

$$
P: \Omega \rightarrow \mathbb{C}_{+}, \quad a \mapsto \infty .
$$

Showing that on the lattice $F_{\epsilon}$ satisfies a discretization of the Riemann BVP (10) and converges to its continuous counterpart is highly non-trivial and a priori not guaranteed - there exist "logical" discretizations of the Boundary Value Problems, whose solutions have degenerate or no scaling limits. We establish convergence in CS09] by considering the primitive $\int_{z_{0}}^{z} F^{2}(u) d u$, which satisfies the 
Dirichlet BVP even in the discrete setting. The big technical problem is that in the discrete case $F^{2}$ is no longer preholomorphic, so its primitive is a priori not preholomorphic or even well-defined. Fortunately, in our setting the imaginary part is still well-defined, so we can set

$$
H_{\epsilon}(z):=\frac{1}{2 \epsilon} \operatorname{Im} \int^{z} F(z)^{2} d z .
$$

While the function $H$ is not exactly preharmonic, it is approximately so, vanishes exactly on the boundary, and is positive inside the domain. This allows to complete the (at times quite involved) proof. A number of non-trivial discrete estimates is called for, and the situation is especially difficult for general isoradial graphs. We provide the needed tools in a separate paper CS08.

Though Theorem 1 establishes convergence of but one observable, the latter (when normalized at $b$ ) is well behaved with respect to the interface traced from $a$. So it can be used to establish the following, see [CS10]:

Corollary 1. As mesh of the lattice tends to zero, the critical Ising interface in the discretization of the domain $\Omega$ with Dobrushin boundary conditions converges to the Schramm's SLE(3) curve.

Convergence is almost immediate in the topology of (probability measures on the space of) Loewner driving functions, but upgrading to convergence of curves requires extra estimates, cf. KS09, DCHN09, CS10. Once interfaces are related to SLE curves, many more properties can be established, including values of dimensions and scaling exponents.

But even without appealing to SLE, one can use preholomorphic functions to a stronger effect. In a joint paper with Hongler [HS10] we study a similar observable, when both ends of the interface are allowed to be inside the domain. It turns out to be preholomorphic in both variables, except for the diagonal, and so its scaling limit can be identified with the Green's function solving the Riemann BVP. On the other hand, when two arguments are taken to be nearby, one retrieves the probability of an edge being present in the contour representation, or that the nearby spins are different. This allows to establish conformal invariance of the energy field in the scaling limit:

Theorem 2 (Hongler, Smirnov). Let $a \in \Omega$ and $\left\langle x^{\epsilon}, y^{\epsilon}\right\rangle$ be the closest edge from $a \in \Omega_{\epsilon}$. Then, as $\epsilon \rightarrow 0$, we have

$$
\begin{aligned}
\mathbb{E}_{+}\left[\sigma_{x}^{\epsilon} \sigma_{y}^{\epsilon}\right] & =\frac{\sqrt{2}}{2}+\frac{l_{\Omega}(a)}{\pi} \cdot \epsilon+o(\epsilon), \\
\mathbb{E}_{\text {free }}\left[\sigma_{x}^{\epsilon} \sigma_{y}^{\epsilon}\right] & =\frac{\sqrt{2}}{2}-\frac{l_{\Omega}(a)}{\pi} \cdot \epsilon+o(\epsilon),
\end{aligned}
$$

where the subscripts + and free denote the boundary conditions and $l_{\Omega}$ is the element of the hyperbolic metric on $\Omega$.

This confirms the Conformal Field Theory predictions and, as far as we know, for the first time provides the multiplicative constant in front of the hyperbolic metric. 


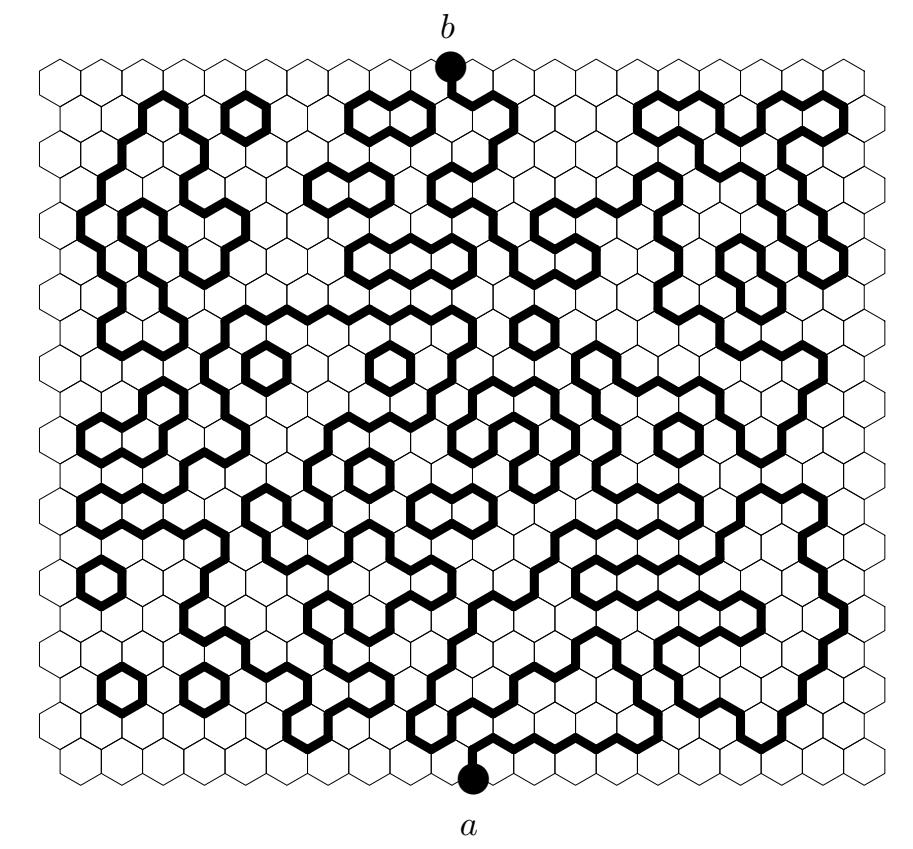

Figure 6. The high-temperature expansion of the $O(N)$ model leads to a gas of disjoint simple loops. Probability of a configuration is proportional to $N^{\# \text { loops }} x^{\text {length }}$. We study it with Dobrushin boundary conditions: besides loops, there is an interface between two boundary points $a$ and $b$.

These techniques were taken further by Hongler in Hon10, where he showed that the (discrete) energy field in the critical Ising model on the square lattice has a conformally covariant scaling limit, which can be then identified with the corresponding Conformal Field Theory. This was accomplished by showing convergence of the discrete energy correlations in domains with a variety of boundary conditions to their continuous counterparts; the resulting limits are conformally covariant and are determined exactly. Similar result was obtained for the scaling limit of the spin field on the domain boundary.

\section{The $O(N)$ model}

The Ising preholomorphic function was introduced in Smi06 in the setting of general $O(N)$ models on the hexagonal lattice. It can be further generalized to a variety of lattice models, see the work of Cardy, Ikhlef, Rajabpour [RC07, IC09. Unfortunately, the observable seems only partially preholomorphic (satisfying only some of the Cauchy-Riemann equations) except for the Ising case. One can make an analogy with divergence-free vector fields, which are not a priori curl-free.

The argument in the previous Section was adapted to the Ising case, and some properties remain hidden behind the notion of the strong holomorphicity. Below we present its version generalized to the $O(N)$ model, following our joint work 
[DCS10] with Duminil-Copin. While for $N \neq 1$ we only prove that our observable is divergence-free, it still turns out to be enough to deduce some global information, establishing the Nienhuis conjecture on the exact value of the connective constant for the hexagonal lattice:

Theorem 3 (Duminil-Copin, Smirnov). On the hexagonal lattice the number $C(k)$ of distinct simple length $k$ curves from the origin satisfies

$$
\lim _{k \rightarrow \infty} \frac{1}{k} \log C(k)=\log \sqrt{2+\sqrt{2}} .
$$

Self-avoiding walks on a lattice (those without self-intersections) were proposed by chemist Flory Flo53 as a model for polymer chains, and turned out to be an interesting and extensively studied object, see the monograph [MS93].

Using Coulomb gas formalism, physicist Nienhuis argued that the connective constant of the hexagonal lattice is equal to $\sqrt{2+\sqrt{2}}$, meaning that (11) holds. He even proposed better description of the asymptotic behavior:

$$
C(k) \approx(\sqrt{2+\sqrt{2}})^{k} k^{11 / 32}, \quad k \rightarrow \infty .
$$

Note that while the exponential term with the connectivity constant is latticedependent, the power law correction is supposed to be universal.

Our proof is partially motivated by Nienhuis' arguments, and also starts with considering the self-avoiding walk as a special case of $O(N)$ model at $N=0$. While a "half-preholomorphic" observable we construct does not seem sufficient to imply conformal invariance in the scaling limit, it can be used to establish the critical temperature, which gives the connective constant.

The general $O(N)$ model is defined for positive integer values of $N$, and is a generalization of the Ising model (to which it specializes for $N=1$ ), with \pm 1 spins replaced by points on a sphere in the $N$-dimensional space. We work with the graphical representation, which is obtained using the high-temperature expansion, and makes the model well defined for all non-negative values of $N$.

We concentrate on the hexagonal lattice in part because it is trivalent and so at most one contour can pass through a vertex, creating no ambiguities. This simplifies the reasoning, though general graphs can also be addressed by introducing additional weights for multiple visits of vertices. We consider configurations $\omega$ of disjoint simple loops on the mesh $\epsilon$ hexagonal lattice inside domain $\Omega_{\epsilon}$, and two parameters: loop-weight $N \geq 0$ and (temperature-like) edge-weight $x>0$. Partition function is then given by

$$
Z=\sum_{\omega} N^{\# \text { loops }} x^{\text {length of contours }} .
$$

A typical configuration is pictured in Figure [6 where we introduced Dobrushin boundary conditions: besides loops, there is an interface $\gamma$ joining two fixed boundary points $a$ and $b$. It was conjectured by Kager and Nienhuis KN04 that in 
the interval $N \in[0,2]$ the model has conformally invariant scaling limits for $x=x_{c}(N):=1 / \sqrt{2+\sqrt{2-N}}$ and $x \in\left(x_{c}(N),+\infty\right)$. The two different limits correspond to dilute/dense regimes, with the interface $\gamma$ conjecturally converging to the Schramm's SLE curves for an appropriate value of $\kappa \in[8 / 3,4]$ and $\kappa \in[4,8]$ correspondingly. The scaling limit for low temperatures $x \in\left(0, x_{c}\right)$ is not conformally invariant.

Note that for $N=1$ we do not count the loops, thus obtaining the lowtemperature expansion of the Ising model on the dual triangular lattice. In particular, the critical Ising corresponds to $x=1 / \sqrt{3}$ by the work Wan50. of Wannier, in agreement with Nienhuis predictions. And for $x=1$ one obtains the critical site percolation on triangular lattice (or equivalently the Ising model at infinite temperature). The latter is conformally invariant in the scaling limit by [Smi01b, Smi01a].

Note also that the Dobrushin boundary conditions make the model well-defined for $N=0$ : then we have only one interface, and no loops. In the dilute regime this model is expected to be in the universality class of the self-avoiding walk.

Analogously to the Ising case, we define an observable (which is now a parafermion of fractional spin) by moving one of the ends of the interface inside the domain. Namely, for an edge center $z$ we set

$$
F_{\epsilon}(z):=\sum_{\omega(a \rightarrow z)} x^{\text {length of contours }} \mathcal{W}(\omega(a \rightarrow z)),
$$

where the sum is taken over all configurations $\omega=\omega(a \rightarrow z)$ which have disjoint simple contours: a number of loops and an interface $\gamma$ joining two exceptional points, $a$ on the boundary and $z$ inside. As before, the sum is corrected by a complex weight with the spin $s \in \mathbb{R}$ :

$$
\mathcal{W}(\omega(a \rightarrow z)):=\exp (-i s \text { winding }(\gamma, a \rightarrow z)),
$$

equivalently we can write

$$
\mathcal{W}(\omega(a \rightarrow z))=\lambda^{\# \text { signed turns of } \gamma}, \quad \lambda:=\exp \left(-i s \frac{\pi}{3}\right) .
$$

Note that on hexagonal lattice one turn corresponds to $\pi / 3$, hence the difference in the definition of $\lambda$.

Our key observation is the following

Lemma 4. For $N \in[0,2]$, set $2 \cos (\theta)=N$ with parameter $\theta \in[0, \pi / 2]$. Then for

$$
\begin{array}{ll}
s=\frac{\pi-3 \theta}{4 \pi}, & x^{-1}=2 \cos \left(\frac{\pi+\theta}{4}\right)=\sqrt{2-\sqrt{2-N},} \text { or } \\
s=\frac{\pi+3 \theta}{4 \pi}, & x^{-1}=2 \cos \left(\frac{\pi-\theta}{4}\right)=\sqrt{2+\sqrt{2-N}},
\end{array}
$$

the observable $F$ satisfies the following relation for every vertex $v$ inside $\Omega_{\epsilon}$ :

$$
(p-v) F(p)+(q-v) F(q)+(r-v) F(r)=0,
$$

where $p, q, r$ are the mid-edges of the three edges adjacent to $v$. 


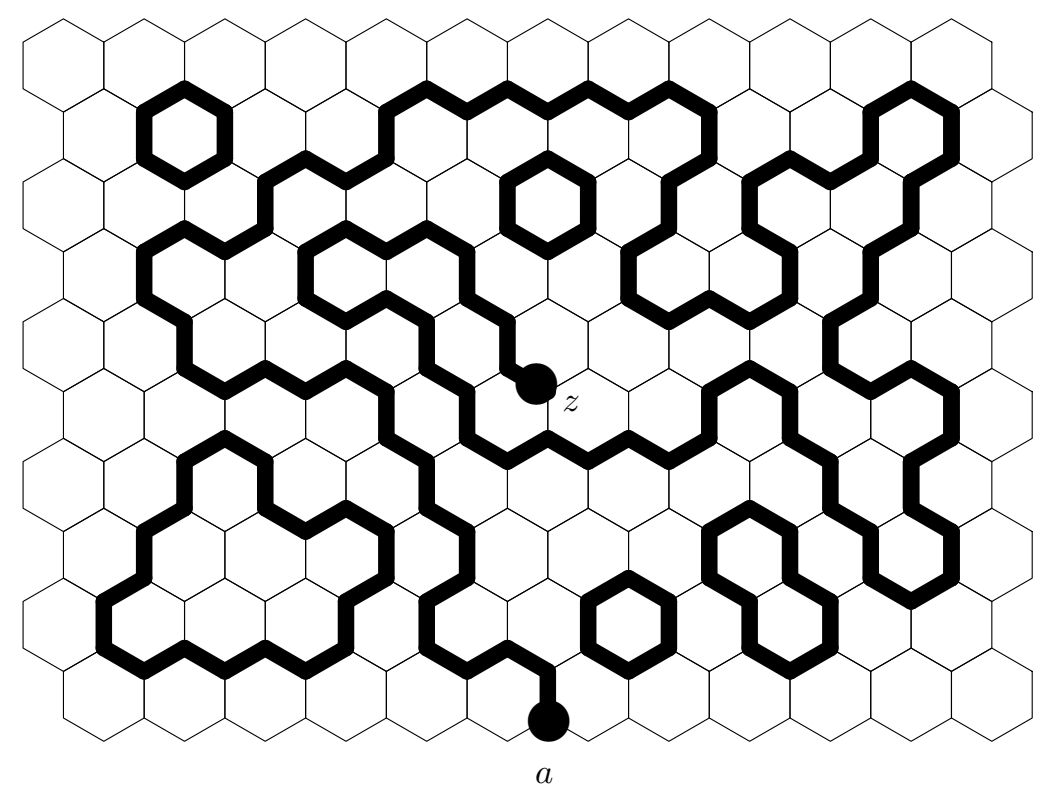

Figure 7. To obtain the parafermionic observable in the $O(N)$ model we consider configurations with an interface joining a boundary point $z$ to an interior point $z$ and weight them by a complex weight depending on the winding of the interface.

Above solution (14) corresponds to the dense, and (15) - to the dilute regime. Note that identity (16) is a form of the first Kirchhoff's law, but apart from the Ising case $N=1$ we cannot verify the second one.

To prove Lemma 4, we note that configurations with an interface arriving at $p, q$ or $r$ can be grouped in triplets, so that three configurations differ only in immediate vicinity of $v$, see Figure 8. It is enough then to check that contributions of three configurations to (16) sum up to zero. But the relative weights of configurations in a triplet are easy to write down as shown in Figure 8 and the coefficients in the identity (16) are proportional to the three cube roots of unity: $1, \tau:=\exp (i 2 \pi / 3)$, $\bar{\tau}$ (if the neighbors of $v$ are taken in the counterclockwise order). Therefore we have to check just two identities:

$$
\begin{aligned}
N+\tau \bar{\lambda}^{4}+\bar{\tau} \lambda^{4} & =0 \\
1+\tau x \bar{\lambda}+\bar{\tau} x \lambda & =0 .
\end{aligned}
$$

Recalling that $\lambda=\exp (-i s \pi / 3)$, the equations above can be recast as

$$
\begin{aligned}
-\frac{2 \pi}{3}-4 s \frac{\pi}{3} & = \pm(\pi-\theta)+2 \pi k, \quad k \in \mathbb{Z}, \\
x & =-1 /\left(2 \cos \left(\frac{(2+s) \pi}{3}\right)\right) .
\end{aligned}
$$



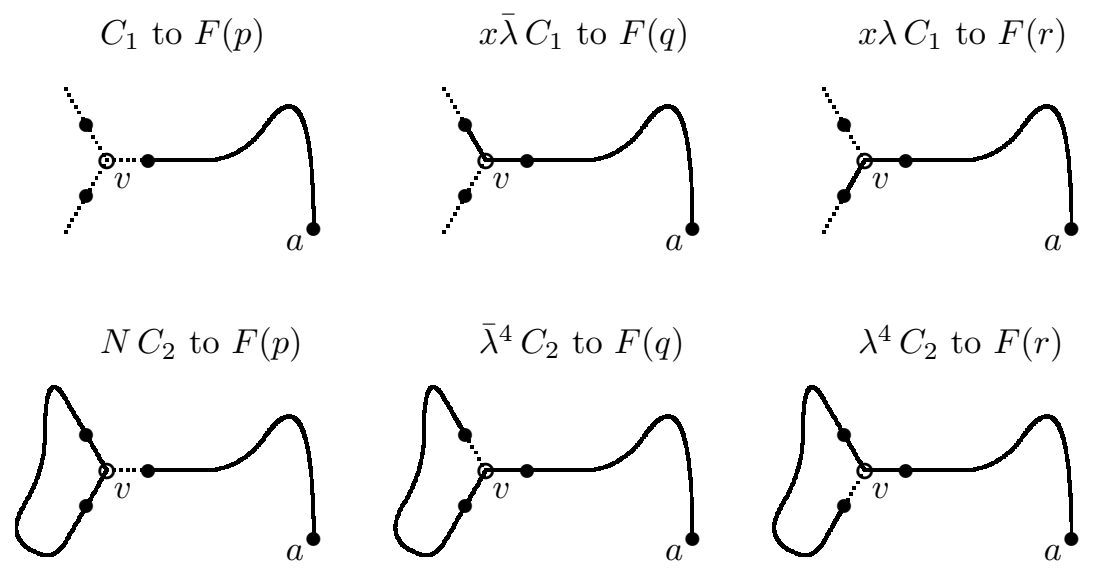

Figure 8. Configurations with the interface ending at one of the three neighbors of $v$ are grouped into triplets by adding or removing half-edges around $v$. Two essential examples of triplets are pictured above, along with their relative contributions to the identity (13).

The first equation implies that

$$
s= \pm\left(-\frac{3}{4}+\frac{3 \theta}{4 \pi}\right)-\frac{1}{2}-\frac{3}{2} k, \quad k \in \mathbb{Z}
$$

and the second equation then determines the allowed value of $x$ uniquely. Most of the solutions of (17) lead to observables symmetric to the two main ones, which are provided by solutions to the equations (14) and (15).

When we set $N=0$, there are no loops, and configurations contain just an interface from $a$ to $z$, weighted by $x^{\text {length }}$. This corresponds to taking $\theta=\pi / 2$ and one of the solutions is given by $s=5 / 8$ and $x_{c}=1 / \sqrt{2+\sqrt{2}}$, as predicted by Nienhuis. To prove his prediction, we observe that summing the identity (16) over all interior vertices implies that

$$
\sum_{z \in \partial \Omega_{\epsilon}} F(z) \eta(z)=0,
$$

where the sum taken over the centers $z$ of oriented edges $\eta(z)$ emanating from the discrete domain $\Omega_{\epsilon}$ into its exterior. Since $F(a)=1$ by definition, we conclude that $F$ for other boundary points sums up to 1 . As in the Ising model, the winding on the boundary is uniquely determined, and (for this particular critical value of $x$ ), one observes that considering the real part of $F$ we can get rid of the complex weights, replacing them by explicit positive constants (depending on the slope of 
the boundary). Thus we obtain an equation

$$
\sum_{z \in \partial \Omega_{\epsilon} \backslash\{a\}} \sum_{\omega(a \rightarrow z)} x_{c}^{\text {length of contours }} \asymp 1,
$$

regardless of the size of the domain $\Omega_{\epsilon}$. A simple counting argument then shows that the series

$$
\sum_{k} C(k) x^{k}=\sum_{\text {simple walks from } a \text { inside } \mathbb{C}} x^{\text {length }},
$$

converges when $x<x_{c}$ and diverges when $x>x_{c}$, clearly implying the conjecture.

Note that establishing the holomorphicity of our observable in the scaling limit would allow to relate self-avoiding walk to the Schramm's SLE with $\kappa=8 / 3$ and together with the work LSW04 of Lawler, Schramm and Werner to establish the more precise form (12) of the Nienhuis prediction.

\section{What's next}

Below we present a list of open questions. As before, we do not aim for completeness, rather we highlight a few directions we find particularly intriguing.

Question 1. As was discussed, discrete complex analysis is well developed for isoradial graphs (or rhombic lattices), see [Duf68, Mer01, Ken02, CS08. Is there a more general discrete setup where one can get similar estimates, in particular convergence of preholomorphic functions to the holomorphic ones in the scaling limit? Since not every topological quadrangulation admits a rhombic embedding KS05, can we always find another embedding with a sufficiently nice version of discrete complex analysis? Same question can be posed for triangulations, with variations of the first definition by Isaacs (5), like the ones in the work of Dynnikov and Novikov DN03 being promising candidates.

Question 2. Variants of the Ising observable were used by Hongler and Kytölä to connect interfaces in domains with more general boundary conditions to more advanced variants of SLE curves, see HK09. Can one use some version of this observable to describe the spin Ising loop soup by a collection of branching interfaces, which converge to a branching SLE tree in the scaling limit? Similar argument os possible for the random cluster representation of the Ising model, see [KS10]. Can one construct the energy field more explicitly than in [Hon10, e.g. in the distributional sense? Can one construct other Ising fields?

Question 3. So far "half-preholomorphic" parafermions similar to ones discussed in this paper have been found in a number of models, see Smi06, RC06, RC07, IC09, but they seem fully preholomorphic only in the Ising case. Can we find the other half of the Cauchy-Riemann equations, perhaps for some modified definition? Note that it seems unlikely that one can establish conformal invariance of the scaling limit operating with only half of the Cauchy-Riemann equations, since there is no conformal structure present. 
Question 4. In the case of the self-avoiding walk, an observable satisfying only a half of the Cauchy-Riemann equations turned out to be enough to derive the value of the connectivity constant DCS10. Since similar observables are available for all other $O(N)$ models, can we use them to establish the critical temperature values predicted by Nienhuis? Our proof cannot be directly transfered, since some counting estimates use the absence of loops. Similar question can be asked for other models.

Question 5. If we cannot establish the preholomorphicity of our observables exactly, can we try to establish it approximately? With appropriate estimates that would allow to obtain holomorphic functions in the scaling limit and hence prove conformal invariance of the models concerned. Note that such more general approach worked for the critical site percolation on the triangular lattice Smi01b, Smi01a, though approximate preholomorphicity was a consequence of exact identities for quantities similar to discrete derivatives.

Question 6. Can we find other preholomorphic observables besides ones mentioned here and in [Smi06]? It is also peculiar that all the models where preholomorphic observables were found so far (the dimer model, the uniform spanning tree, the Ising model, percolation, etc.) can be represented as dimer models. Are there any models in other universality classes, admitting a dimer representation? Can then Kenyon's techniques Ken04, Ken09] be used to find preholomorphic observables by considering the Kasteleyn's matrix and the coupling function?

Question 7. Throughout this paper we were concerned with linear discretizations of the Cauchy-Riemann equations. Those seem more natural in the probabilistic context, in particular they might be easier to relate to the SLE martingales, cf. Smi06. However there are also well-known non-linear versions of the CauchyRiemann equations. For example, the following version of the Hirota equation for a complex-valued function $F$ arises in the context of the circle packings, see e.g. [BMS05:

$$
\frac{(F(z+i \epsilon)-F(z-\epsilon))(F(z-i \epsilon)-F(z+\epsilon))}{(F(z+i \epsilon)-F(z+\epsilon))(F(z-i \epsilon)-F(z-\epsilon))}=-1 .
$$

Can we observe this or a similar equation in the probabilistic context and use it to establish conformal invariance of some model? Note that plugging into the equation (18) a smooth function, we conclude that to satisfy it approximately it must obey the identity

$$
\left(\partial_{x} F(z)\right)^{2}+\left(\partial_{y} F(z)\right)^{2}=0 .
$$

So in the scaling limit (18) can be factored into the Cauchy-Riemann equations and their complex conjugate, thus being in some sense linear. It does not seem possible to obtain "essential" non-linearity using just four points, but using five points one can create one, as in the next question.

Question 8. A number of non-linear identities was discovered for the correlation functions in the Ising model, starting with the work of Groeneveld, Boel and 
Kasteleyn GBK78, BK78. We do not want to analyze the extensive literature to-date, but rather pose a question: can any of these relations be used to define discrete complex structures and pass to the scaling limit? In two of the early papers by McCoy, Wu and Perk MW80, Per80, a quadratic difference relation was observed in the full plane Ising model first on the square lattice, and then on a general graph. To better adapt to our setup, we rephrase this relation for the correlation $C(z)$ of two spins (one at the origin and another at $z$ ) in the Ising model at criticality on the mesh $\epsilon$ square lattice. In the full plane, one has

$$
C(z+i \epsilon) C(z-i \epsilon)+C(z+\epsilon) C(z-\epsilon)=2 C(z)^{2} .
$$

Note that $C$ is a real-valued function, and the equation (19) is a discrete form of the identity

$$
C(z) \Delta C(z)+|\nabla C(z)|^{2}=0 .
$$

The latter is conformally invariant, and is solved by moduli of analytic functions. Can one write an analogous to (19) identity in domains with boundary, perhaps approximately? Can one deduce conformally invariant scaling limit of the spin correlations in that way?

Question 9. Recently there was a surge of interest in random planar graphs and their scaling limits, see e.g. [DS09, LGP08. Can one find observables on random planar graphs (weighted by the partition function of some lattice model) which after an appropriate embedding (e.g. via a circle packing or a piecewise-linear Riemann surface) are preholomorphic? This would help to show that planar maps converge to the Liouville Quantum Gravity in the scaling limit.

Question 10. Approach to the two-dimensional integrable models described here is in several aspects similar to the older approaches based on the Yang-Baxter relations Bax89. Some similarities are discussed in Cardy's paper Car09. Can one find a direct link between the two approaches? It would also be interesting to find a link to the three-dimensional consistency relations as discussed in [BMS09.

Question 11. Recently Kenyon investigated the Laplacian on the vector bundles over graphs in relation to the spanning trees [Ken10]. Similar setup seems natural for the Ising observable we discuss. Can one obtain more information about the Ising and other models by studying difference operators on vector bundles over the corresponding graphs?

Question 12. Can anything similar be done for the three-dimensional models? While preholomorphic functions do not exist here, preharmonic vector fields are well-defined and appear naturally for the Uniform Spanning Tree and the Loop Erased Random Walk. To what extent can they be used? Can one find any other difference equations in three-dimensional lattice models?

\section{References}

[Bax78] R. J. Baxter. Solvable eight-vertex model on an arbitrary planar lattice. Philos. Trans. Roy. Soc. London Ser. A, 289(1359):315-346, 1978. 
[Bax89] Rodney J. Baxter. Exactly solved models in statistical mechanics. Academic Press Inc. [Harcourt Brace Jovanovich Publishers], London, 1989. Reprint of the 1982 original.

[BdT08] Cédric Boutillier and Béatrice de Tilière. The critical Z-invariant Ising model via dimers: the periodic case. Probab. Theory Related Fields, 147: 379-413, 2010 .

[BdT09] Cédric Boutillier and Béatrice de Tilière. The critical Z-invariant Ising model via dimers: locality property. Comm. Math. Physics, to appear. Preprint, arXiv:0902.1882, 2009.

[BK78] R. J. Boel and P. W. Kasteleyn. Correlation-function identities and inequalities for Ising models with pair interactions. Comm. Math. Phys., 61(3):191208, 1978.

[BMS05] Alexander I. Bobenko, Christian Mercat, and Yuri B. Suris. Linear and nonlinear theories of discrete analytic functions. Integrable structure and isomonodromic Green's function. J. Reine Angew. Math., 583:117-161, 2005.

[BMS09] Vladimir V. Bazhanov, Vladimir V. Mangazeev, and Sergey M. Sergeev. Quantum geometry of 3-dimensional lattices and tetrahedron equation. Preprint, arXiv:0911.3693, 2009.

[Bou26] George Bouligand. Sur le problemè de Dirichlet. Ann. Soc. Pol. Math., 4:59$112,1926$.

[BS08] Alexander I. Bobenko and Yuri B. Suris. Discrete differential geometry, volume 98 of Graduate Studies in Mathematics. American Mathematical Society, Providence, RI, 2008.

[BSST40] R. L. Brooks, C. A. B. Smith, A. H. Stone, and W. T. Tutte. The dissection of rectangles into squares. Duke Math. J., 7:312-340, 1940.

[Car09] John Cardy. Discrete holomorphicity at two-dimensional critical points. J. Stat. Phys., 137(5-6):814-824, 2009.

[CFL28] R. Courant, K. Friedrichs, and H. Lewy. Über die partiellen Differenzengleichungen der mathematischen Physik. Math. Ann., 100:32-74, 1928.

[CS08] Dmitry Chelkak and Stanislav Smirnov. Discrete complex analysis on isoradial graphs. Adv. in Math., to appear. Preprint, arXiv:0810.2188, 2008.

[CS09] Dmitry Chelkak and Stanislav Smirnov. Universality in the 2D Ising model and conformal invariance of fermionic observables. Invent. Math., to appear. Preprint, arXiv:0910.2045, 2009.

[CS10] Dmitry Chelkak and Stanislav Smirnov. Conformal invariance of the 2D Ising model at criticality. Preprint, 2010.

[DCHN09] Hugo Duminil-Copin, Clément Hongler, and Pierre Nolin. Connection probabilities and RSW-type bounds for the FK Ising model. Preprint, arXiv:0912.4253, 2009.

[DCS10] Hugo Duminil-Copin and Stanislav Smirnov. The connective constant of the honeycomb lattice equals $\sqrt{2+\sqrt{2}}$. Preprint, arXiv:1007.0575, 2010.

[DM71] Charles R. Deeter and C. Wayne Mastin. The discrete analog of a minimum problem in conformal mapping. Indiana Univ. Math. J., 20:355-367, 1970/1971. 
[DN03] I. A. Dynnikov and S. P. Novikov. Geometry of the triangle equation on two-manifolds. Moscow Math. J., 3:419-438, 2003.

[DS09] Bertrand Duplantier and Scott Sheffield. Duality and the Knizhnik-PolyakovZamolodchikov relation in Liouville quantum gravity. Phys. Rev. Lett., 102(15):150603, 4, 2009.

[Duf56] R. J. Duffin. Basic properties of discrete analytic functions. Duke Math. J., 23:335-363, 1956.

[Duf68] R. J. Duffin. Potential theory on a rhombic lattice. J. Combinatorial Theory, 5:258-272, 1968.

[Fer44] Jacqueline Ferrand. Fonctions préharmoniques et fonctions préholomorphes. Bull. Sci. Math. (2), 68:152-180, 1944.

[Flo53] P. Flory. Principles of Polymer Chemistry. Cornell University Press, 1953.

[GBK78] J. Groeneveld, R. J. Boel, and P. W. Kasteleyn. Correlation-function identities for general planar Ising systems. Physica A: Statistical and Theoretical Physics, 93(1-2):138-154, 1978.

[HK09] Clément Hongler and Kalle Kytölä. Dipolar SLE in Ising model with plusminus-free boundary conditions. Preprint, 2009.

[Hon10] Clément Hongler. Conformal invariance of the Ising model correlations. Ph.D. thesis, Universté de Genève, 2010.

[HS93] Zheng-Xu He and Oded Schramm. Fixed points, Koebe uniformization and circle packings. Ann. of Math. (2), 137(2):369-406, 1993.

[HS10] Clément Hongler and Stanislav Smirnov. Energy density in the 2D Ising model. Preprint, arXiv:1008.2645, 2010.

[IC09] Yacine Ikhlef and John Cardy. Discretely holomorphic parafermions and integrable loop models. J. Phys. A, 42(10):102001, 11, 2009.

[Isa41] Rufus Philip Isaacs. A finite difference function theory. Univ. Nac. Tucumán. Revista A., 2:177-201, 1941.

[Isa52] Rufus Isaacs. Monodiffric functions. Construction and applications of conformal maps. In Proceedings of a symposium, National Bureau of Standards, Appl. Math. Ser., No. 18, pages 257-266, Washington, D. C., 1952. U. S. Government Printing Office.

[KC71] Leo P. Kadanoff and Horacio Ceva. Determination of an operator algebra for the two-dimensional Ising model. Phys. Rev. B (3), 3:3918-3939, 1971.

[Ken00] Richard Kenyon. Conformal invariance of domino tiling. Ann. Probab., 28(2):759-795, 2000 .

[Ken01] Richard Kenyon. Dominos and the Gaussian free field. Ann. Probab., 29(3):1128-1137, 2001.

[Ken02] Richard Kenyon. The Laplacian and Dirac operators on critical planar graphs. Invent. Math., 150(2):409-439, 2002.

[Ken04] Richard Kenyon. An introduction to the dimer model. In School and Conference on Probability Theory, ICTP Lect. Notes, XVII, pages 267-304 (electronic). Abdus Salam Int. Cent. Theoret. Phys., Trieste, 2004. 
[Ken09] Richard Kenyon. Lectures on dimers. In Statistical mechanics, volume 16 of IAS/Park City Math. Ser., pages 191-230. Amer. Math. Soc., Providence, RI, 2009.

[Ken10] Richard Kenyon. Spanning forests and the vector bundle Laplacian. Preprint arXiv:1001.4028, 2010.

[Kir47] G. Kirchhoff. Ueber die Aufösung der Gleichungen, auf welche man bei der Untersuchung der linearen Vertheilung galvanischer Ströme geführt wird. Annalen der Physik und Chemie, 148(12):497-508, 1847.

[Kis05] Christer O. Kiselman. Functions on discrete sets holomorphic in the sense of Isaacs, or monodiffric functions of the first kind. Sci. China Ser. A, 48(suppl.):86-96, 2005.

[KN04] Wouter Kager and Bernard Nienhuis. A guide to stochastic Löwner evolution and its applications. J. Statist. Phys., 115(5-6):1149-1229, 2004.

[KS05] Richard Kenyon and Jean-Marc Schlenker. Rhombic embeddings of planar quad-graphs. Trans. Amer. Math. Soc., 357(9):3443-3458 (electronic), 2005.

[KS09] Antti Kemppainen and Stanislav Smirnov. Random curves, scaling limits and Loewner evolutions. Preprint, University of Helsinki, 2009.

[KS10] Antti Kemppainen and Stanislav Smirnov. Conformal invariance in random cluster models. III. Full scaling limit. In preparation, 2010.

[KW41] H.A. Kramers and G.H. Wannier. Statistics of the two-dimensional ferromagnet. Pt. 1. Phys. Rev., II. Ser., 60:252-262, 1941.

[LF55] Jacqueline Lelong-Ferrand. Représentation conforme et transformations à intégrale de Dirichlet bornée. Gauthier-Villars, Paris, 1955.

[LGP08] Jean-François Le Gall and Frédéric Paulin. Scaling limits of bipartite planar maps are homeomorphic to the 2-sphere. Geom. Funct. Anal., 18(3):893-918, 2008.

[Lov04] László Lovász. Discrete analytic functions: an exposition. In Surveys in differential geometry. Vol. IX, Surv. Differ. Geom., IX, pages 241-273. Int. Press, Somerville, MA, 2004.

[LSW04] Gregory F. Lawler, Oded Schramm, and Wendelin Werner. On the scaling limit of planar self-avoiding walk. In Fractal geometry and applications: a jubilee of Benoît Mandelbrot, Part 2, volume 72 of Proc. Sympos. Pure Math., pages 339-364. Amer. Math. Soc., Providence, RI, 2004.

[Lus26] L. Lusternik. Uber einege Anwendungen der direkten Methoden in Variationsrechnung. Recueil de la Société Mathématique de Moscou, pages 173-201, 1926.

[Mer01] Christian Mercat. Discrete Riemann surfaces and the Ising model. Comm. Math. Phys., 218(1):177-216, 2001.

[Mer07] Christian Mercat. Discrete Riemann surfaces. In Handbook of Teichmüller theory. Vol. I, volume 11 of IRMA Lect. Math. Theor. Phys., pages 541-575. Eur. Math. Soc., Zürich, 2007.

[MS93] Neal Madras and Gordon Slade. The self-avoiding walk. Probability and its Applications. Birkhäuser Boston Inc., Boston, MA, 1993. 
[MS09] Nikolai Makarov and Stanislav Smirnov. Off-critical lattice models and massive SLEs. In XIVth International Congress on Mathematical Physics, Lisbon, Portugal, July 28 - August 2, 2003, pages 362-371. World Sci. Publ., Singapore, 2009.

[MW80] Barry M. McCoy and Tai Tsun Wu. Non-linear partial difference equations for the two-dimensional Ising model. Physics Review Letters, 45:675-678, 1980.

[Per80] J. H. H. Perk. Quadratic identities for Ising model correlations. Physics Letters, 79A:3-5, 1980.

[PW23] H. B. Phillips and N. Wiener. Nets and the Dirichlet problem. Mass. J. of Math., 2:105-124, 1923.

[RC06] V. Riva and J. Cardy. Holomorphic parafermions in the Potts model and stochastic Loewner evolution. J. Stat. Mech. Theory Exp., (12):P12001, 19 pp. (electronic), 2006.

[RC07] M. A. Rajabpour and J. Cardy. Discretely holomorphic parafermions in lattice $Z_{N}$ models. J. Phys. A, 40(49):14703-14713, 2007.

[RS87] Burt Rodin and Dennis Sullivan. The convergence of circle packings to the Riemann mapping. J. Differential Geom., 26(2):349-360, 1987.

[Smi01a] Stanislav Smirnov. Critical percolation in the plane. Preprint, arXiv:0909.4499, 2001.

[Smi01b] Stanislav Smirnov. Critical percolation in the plane: Conformal invariance, Cardy's formula, scaling limits. C. R. Math. Acad. Sci. Paris, 333(3):239-244, 2001.

[Smi06] Stanislav Smirnov. Towards conformal invariance of 2D lattice models. SanzSolé, Marta (ed.) et al., Proceedings of the international congress of mathematicians (ICM), Madrid, Spain, August 22-30, 2006. Volume II: Invited lectures, 1421-1451. Zürich: European Mathematical Society (EMS), 2006.

[Smi10] Stanislav Smirnov. Conformal invariance in random cluster models. I. Holomorphic spin structures in the Ising model. Ann. of Math. (2), 172:101-133, 2010.

[Ste05] Kenneth Stephenson. Introduction to circle packing. The theory of discrete analytic functions. Cambridge University Press, Cambridge, 2005.

[Thu86] William P. Thurston. Zippers and univalent functions. In The Bieberbach conjecture (West Lafayette, Ind., 1985), volume 21 of Math. Surveys Monogr., pages 185-197. Amer. Math. Soc., Providence, RI, 1986.

[Wan50] G. H. Wannier. Antiferromagnetism. The triangular Ising net. Phys. Rev., 79(2):357-364, Jul 1950.

Section de Mathématiques, Université de Genève. 2-4 rue du Lièvre, CP 64, 1211

Genève 4, SUISSE

E-mail: stanislav.smirnov@unige.ch 